\title{
Estudo Teórico do Efeito de Substituintes Doadores e Aceptores de Densidade Eletrônica na Síntese da Acetanilida
}

\author{
Karine N. de Andrade, Daniel G. S. Quattrocioccchi \\ \& Leonardo M. da Costa
}

Foi realizado um estudo DFT (B3LYP/6-311++G(d,p)) da influência do efeito de substituintes doadores e aceptores de densidade eletrônica na síntese da acetanilida. Os substituintes foram posicionados tanto na molécula de anidrido, quanto na anilina. A análise quântica foi realizada por meio do cálculo de $\Delta \mathrm{H}$ e $\Delta \mathrm{G} 298$ da reação. Posteriormente os resultados foram apresentados em aulas experimentais de graduação em química/farmácia e engenharia química para verificação da aprendizagem.

Palavras Chave: DFT; Efeito do substituinte; Ensino de química; Acetanilida.

A DFT (B3LYP/6-311++G(d,p)) study of the influence of the electron donor or accepting effect of substituents on the acetanilide synthesis was performed. The substituents were positioned both in the anhydride and the anilide reactants. The quantum analysis was performed by the calculation of the reaction $\Delta \mathrm{H}$ e $\Delta \mathrm{G} 298$. Additionally, the results were presented in experimental classes of chemistry, farmacy and chemical engeneering undergratuation courses to verify the mode learning.

Keywords: DFT, Substituent effect; Chemistry teaching; Acetanilide. 


\section{Introdução}

A reação de síntese da acetanilida tem sido muito utilizada nos laboratórios de ensino de química orgânica devido à facilidade de manipulação dos equipamentos e reagentes. Também apresenta baixo custo para realização e seu mecanismo está bem elucidado na literatura. ${ }^{1}$ A acetanilida é utilizada como intermediário reacional em diversas sínteses industriais e na calibração de instrumentos (ponto de fusão e aparelhos de análise térmica)1, além ser de ser um dos precursores do paracetamol (analgésico e antitérmico). ${ }^{2}$ Diversas sínteses tem sido propostas com o objetivo de aumentar a eficiência da reação. A modelagem molecular auxilia nos estudos de reações orgânicas para a otimização de condições reacionais que levem ao aumento de rendimento. ${ }^{3}$ Este trabalho visa avaliar o efeito de substituintes posicionados no anidrido acético e na anilina na modulação de $\Delta \mathrm{H}$ e $\Delta \mathrm{G}^{298}$ da síntese da acetanilida (Figura 1).

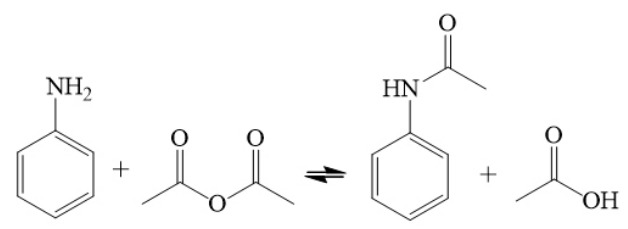

Figura 1. Reação de síntese da Acetanilida.

Outro importante tópico nesse trabalho é proporcionar na aula experimental da síntese da acetanilida a abordagem de efeitos eletrônicos. A partir dos resultados de $\Delta \mathrm{H}$ e $\Delta \mathrm{G} 298$ obtidos o professor pode correlaciona-los com conceitos de estruturas de ressonância, ataque nucleofílico, efeitos indutivo e mesomérico.

\section{Metodologia}

Foram realizados cálculos de otimização de estrutura com o funcional B3LYP e o conjunto base 6-311++G(d,p). Após, foi realizado o cálculo de frequências vibracionais para obter as correções energéticas de entalpia e de energia livre de Gibbs para o calor da reação $(\Delta \mathrm{E})$ apresentada na Figura 1. Essa associação de funcional e conjunto base gera resultados em concordância com métodos $a b$ initio mais robustos.4 Os efeitos eletrônicos (indução e ressonância) dos substituintes na síntese da acetanilida foram caracterizados através da variação de grupamentos doadores e aceptores de densidade eletrônica nas moléculas de anidrido (Figura 2) e de anilina (Figura 3). Foi avaliado a influencia desses substituintes na modulação de $\Delta \mathrm{H}$ e de $\Delta \mathrm{G}^{298}$ da reação.<smiles>[R]C(=O)OC([R])=O</smiles>

Figura 2. Molécula de anidrido com os seguintes grupos R substituintes: $-\mathrm{CF}_{3}$ e $-\mathrm{CN}$ (elétron aceptores); $-\mathrm{CH}=\mathrm{CH}-\mathrm{OH}$ e $-\mathrm{CH}=\mathrm{CH}-\mathrm{OCH} 3$ (elétron doadores). (a)<smiles>[R]c1ccc(N)cc1</smiles>

(b)<smiles>[R]c1ccccc1N</smiles>

Figura 3. Molécula de anilina, (a) para substituída $(\mathrm{R}=-\mathrm{OCH} 3,-\mathrm{CN}$ -NO2) e (b) orto substituída ( $\mathrm{R}=$ t-butil).

Durante uma aula experimental de síntese da acetanilida na Universidade Federal Fluminense foi explicada a modulação dos valores de $\Delta \mathrm{H}$ e $\Delta \mathrm{G} 298$ mediante a alteração dos substituintes. Após, foi realizado um questionário diagnóstico para avaliação da inserção dos conteúdos (estrutura de ressonância, ataque nucleofílico e efeitos eletrônicos) na aula.

\section{Resultados e Discussão}

Os valores de $\Delta H$ e $\Delta G 298$ são apresentados na tabela 1. A análise da tabela mostra que a reação é favorecida ( $\Delta \mathrm{H}$ e $\Delta \mathrm{G} 298$ mais negativos do que na reação padrão) por dois fatores: (1) posicionamento de substituintes elétrons aceptores no anidrido, que aumentam a eletrofilicidade do 
carbono carbonílico, deixando-o mais susceptível ao ataque nucleofílico; (2) presença de substituintes doadores de elétrons na anilina, que aumentam a densidade eletrônica sob o átomo de nitrogênio, tornando-o mais nucleofílico, favorecendo a ocorrência da reação.

Tabela 1. Valores de $\Delta H$ e $\Delta \mathrm{G} 298$, em kcal.mol-1, para a reação de síntese da acetanilida partindo de reagentes substituídos.

\begin{tabular}{|c|c|c|}
\hline $\mathrm{R}$ & $\Delta \mathrm{H}$ & $\Delta \mathrm{G} 298$ \\
\hline$-\mathrm{CH} 3$ (padrão) & -12.33 & -10.96 \\
\hline \multicolumn{3}{|c|}{ Substituintes no anidrido } \\
\hline$-\mathrm{CF} 3$ & -20.86 & -22.34 \\
\hline$-\mathrm{CN}$ & -22.34 & -21.51 \\
\hline$-\mathrm{CH}=\mathrm{CH}-\mathrm{OH}$ & -6.71 & -6.07 \\
\hline$-\mathrm{CH}=\mathrm{CH}-\mathrm{OCH} 3$ & -7.00 & -6.00 \\
\hline \multicolumn{3}{|c|}{ Substituintes na aniline } \\
\hline -t-butil orto & -17.07 & -17.75 \\
\hline -OCH3 para & -13.91 & -14.83 \\
\hline -CN para & -6.59 & -6.01 \\
\hline -NO2 para & -5.90 & -5.34 \\
\hline
\end{tabular}

O posicionamento de grupos aceptores de densidade eletrônica no anidrido torna o $\Delta \mathrm{H}$ e o $\Delta \mathrm{G} 298$ mais negativo em $9.27 \pm 1.05 \mathrm{kcal}^{\mathrm{mol}}{ }^{-1} \mathrm{e} 10.96 \pm 0.60 \mathrm{kcal} . \mathrm{mol}^{-1}$, respectivamente, em relação à reação padrão. A presença de substituintes doadores de elétrons na anilina torna o $\Delta \mathrm{H}$ e o $\Delta \mathrm{G} 298$ mais negativo em $3.16 \pm 2.23 \mathrm{kcal}^{\mathrm{mol}}{ }^{-1}$ e em $2.23 \pm 2.06 \mathrm{kcal}^{\mathrm{mol}}{ }^{-1}$, respectivamente, em relação à reação padrão.

Os substituintes estudados que apresentam $\Delta \mathrm{G} 298$ mais negativo para o anidrido e para a anilina são apresentados, respectivamente, na Figura 4.

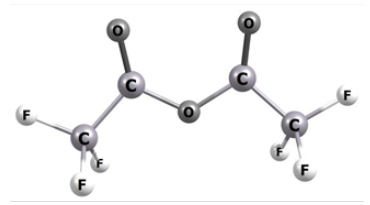

(a)

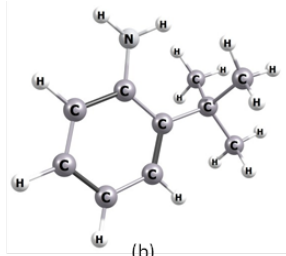

(b)
Figura 4. Estruturas otimizadas das moléculas que apresentam maior espontaneidade (a) anidrido substituído por-CF3 e (b) anilina substituída por t-butil.
O resultado do questionário diagnose realizado é mostrado na Figura 5.

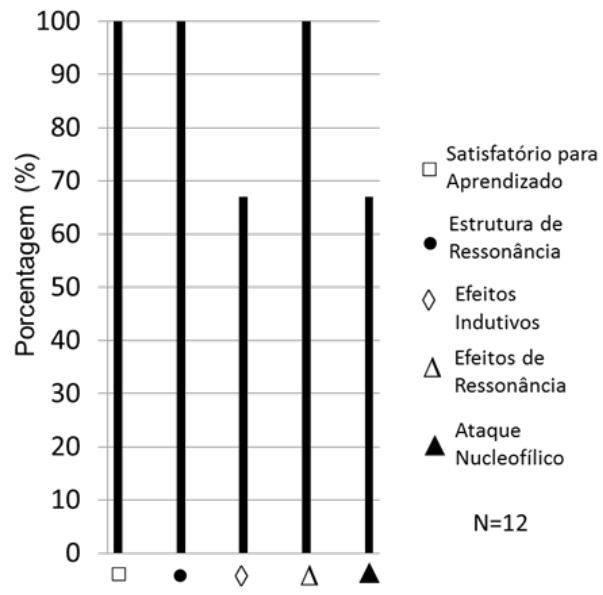

Figura 5. Resultado do questionário diagnóstico.

A análise da Fig. 5 mostra que a discussão dos efeitos dos substituintes na reação de síntese da acetanilida foi satisfatória para o aprendizado de estruturas e efeitos de ressonância, além de efeito indutivo e ataque nucleofílico.

\section{Conclusões}

Com base nos valores obtidos conclui-se que todas as reações estudadas apresentaram valores de $\Delta \mathrm{H}$ e $\Delta \mathrm{G} 298$ negativos, o que demonstra que as reações são exotérmicas e espontâneas. Além disso, substituintes elétron aceptores no anidrido e elétron doadores na anilina favorecem o ataque nucleofílico e assim aumentam a espontaneidade da reação. No questionário diagnóstico realizado observase que a correlação entre a variação dos substituintes nas moléculas reagentes com a modulação de $\Delta \mathrm{H}$ e $\Delta \mathrm{G} 298$ foi significativa para o aprendizado.

\section{Agradecimentos}

Agradecemos aos órgãos de fomento FAPERJ, CAPES, CNPq e PROGRAD. 


\section{Referências Bibliográficas}

1. Cunha, S. et al. Acetanilida: síntese verde sem solvente. Revista Química Nova, 38, 874-876, 2015.

2. Kianmehr, A. et al. Palladium-Catalyzed Regioselctive Direct Cyanation of Acetanilide Derivates with $\mathrm{K} 4[\mathrm{Fe}(\mathrm{CN}) 6]$ by C-H Bond Activation. European Journal of Organic Chemistry.25, 4269-4274, 2016.

3. Mirjafary, Z. et al. Efficient sysnthesis of novel pyranoquinoline derivatives from simple acetanilide derivatives: experimental and theoretical study of their physicochemical properties using DFT calculations. Journal of the Brazilian Chemical Society, 25, 7, 1253 1260, 2014.

4. Corral, I. et al. Agostic vs $\pi$-Interactions in Complexes of Ethynylsilanes and Ethynylgermanes with $\mathrm{Cu}+$ in the Gas Phase The Journal of Physical Chemistry A, 107, 1370-1376, 2003.

\section{Karine N. de Andrade ${ }^{\text {* }}$, Daniel G. S. Quattrocioccchi ${ }^{2}$ \& Leonardo M. da Costa ${ }^{2}$}

${ }^{1}$ Departamento de Química Orgânica, Universidade Federal Fluminense, Outeiro de São João Batista s/n 24020-141 Niterói, RJ, Brasil.

${ }^{2}$ Programa de Pós-graduação em Química, Instituto de Química, Universidade Federal Fluminense, Outeiro de São João Batista s/n 24020141 Niterói, RJ, Brasil.

"E-mail: Karine-andrade@outlook.com 